\title{
Cross-Cultural Adaptation, Validity and Reliability of the Igbo Version of the Brief Fear of Movement Scale for Osteoarthritis
}

\author{
Ezinne Ekediegwu ( $\nabla$ tshantec@yahoo.com ) \\ Ezugwu Uchechukwu Anthonia \\ University of Nigeria Faculty of Health Sciences and Technology \\ Uchenwoke Chigozie Ikenna \\ University of Nigeria Faculty of Health Sciences and Technology \\ Tabansi-Ochiogu Chidinma \\ University of Nigeria Faculty of Health Sciences and Technology \\ Esther Obidike \\ University of Nigeria Faculty of Health Sciences and Technology
}

Nnamdi Azikiwe University Faculty of Health Science and Technology https://orcid.org/0000-0002-2520-8487

Research

Keywords: Igbo, fear, movement, knee, osteoarthritis

Posted Date: November 9th, 2021

DOI: https://doi.org/10.21203/rs.3.rs-1005519/v1

License: @ (i) This work is licensed under a Creative Commons Attribution 4.0 International License. Read Full License 


\section{Abstract}

Background; the brief fear of movement scale for knee osteoarthritis (b-FMSKOA) is a valid instrument for assessing fear of movement in individuals with OA. The objective of this study was to translate and culturally adapt the b-FMSKOA into Igbo language (b-FMSKO-I) and assess its validity and reliability in patients with knee osteoarthritis to promote its use in Igbo speaking parts of Nigeria.

Methods; the original b-FMSKOA was translated and culturally adapted from English into Igbo following a standardized procedure according to Beaton et al., 2000 in seven separate processes of translation, back translation, committee review and pre-testing before being tested for psychometric quality. It was administered (within a 2day interval) to 15 consenting outpatients with knee OA who attended physiotherapy unit 3times a week in a selected South-eastern Nigerian hospital. The b-FMSKOA (English version) was correlated with the lgbo version.

Results; the concurrent validity of the b-FMSKOA-I was found to be satisfactory and comparable to the original version. The correlation coefficient of the variables of interest were within the range of 0.441 and 0.998 and all showed significant correlation $(p<0.05)$ between the scores obtained from the English and Igbo version of the questionnaire except for the fourth item $(r=0.441 ; p=0.131)$ which showed no significant relationship. Cronbach's alpha showed the questionnaire to reach acceptable reliability $a=0.85$. The Pearson correlation coefficient of the variables of interest was within the range of 0.441 and 0.945 with the fourth and first item recording the lowest $(r=0.441)$ and highest $(r=0.945)$ correlation coefficient respectively. All variables showed significant correlation $(p<0.05)$ between the scores obtained from Igbo version of the questionnaires except for the fourth item $(r=0.441 ; p=0.131)$ which showed no significant relationship

Conclusion; the b-FMSKO-I like the original version is a reliable, consistent and valid instrument that can be considered for use in Southeastern Nigeria for evidence based quality healthcare promotion in patients with knee osteoarthritis.

\section{Introduction}

Knee osteoarthritis (KOA) is the most common musculoskeletal disease among older adults, especially women $\geq 50$ years (1). It is a slow, progressive deterioration of synovial membrane of articular cartilage in the knee, causing the people to have knee pain and physical disability (2).Fear of movement, or kinesiophobia, is described as debilitating fear of physical movement and activity that an individual believes will cause pain, injury, or re-injury (3). In patients with osteoarthritis (OA), maintaining a sufficient level of daily activity is critical to managing pain and disability associated with the disease (4), although individuals who experience fear of movement maybe hesitant to initiate or engage in daily activity. Several studies have shown the negative impact of fear of movement on individuals with osteoarthritis. In a recent study (5) in knee osteoarthritis, fear of movement was significantly associated with higher psychological disability and slower gait speed after accounting for some demographic and medical variables. The Brief Fear of Movement Scale (6) was therefore developed to assess fear of movement in individuals with OA with its original version in English.

The translation and cultural adaptation of instruments is an internationally recognized method (7). Translation involves obtaining a version that is semantically equivalent to the original while cross-cultural adaptation is necessary when the instrument is intended for use on a target population that is culturally different from that of the original version.

Nigeria is a multi-ethnic country of over 500 indigenous languages however with Igbo being one of the three major ones (8). English is the official language of communication in Nigeria but however, it's been reported that a sizeable number of patients in Nigeria do not speak or write English (9). There is therefore a need to translate the brief fear of movement scale for knee osteoarthritis (b-FMSKO) into the at least one of the major indigenous languages of Nigeria for cross-cultural comparisons of outcome assessment and to facilitate its use among a certain group of patients. Hence the objective of this study was to translate and cross-culturally adapt the b-FMSO into lgbo (b-FMSO-I) (and subsequently to other major indigenous Nigerian languages) and to study its psychometric properties in patients with knee OA. This will promote the use of b-FMSKO in the Igbo speaking population of Nigeria and ensure that Igbo-speaking patients who do not speak or understand English are not excluded from assessment using b-FMSKO.

\section{Methods}

The study consisted of two stages. First, the b-FMSKOA was translated into lgbo language following a standardized procedure (Beaton, Bombardier, Guillemin, \& Ferraz, 2000). Secondly, the final translated version was tested for psychometric properties. Copies of the Igbo version were completed by consenting outpatients with knee OA who attended physiotherapy unit in a selected South-easternstern Nigerian hospital. 


\section{Translation}

The questionnaire was translated according to the guidelines for cross-cultural adaptation of self-report measures by Beaton et al. (2000). The translation was in seven phases viz;

Forward translation by two bilingual translators, synthesis, back-translation, committee review (first), back-translation by two bilingual translators (English). This was followed by a second committee review/ evaluation by a team of experts consisting of forward and backward translators and two physiotherapists and finally pre-testing in a group of 5 patients who followed a rehabilitation program for knee osteoarthritis at the outpatient physiotherapy department of Nnamdi Azikiwe University Teaching hospital, Anambra, Southeasternstern Nigeria\{figure 1\}.

None of the patients had problems with the translation of any of the questions as the expert committee made sure the sentence structures were clear and unambiguous. The authors can provide a detailed report of the translation process. For the final version of the b-FMSKOA-I, see appendix A.

\section{Ethical Consideration}

Prior to the commencement of this study, ethical approval was sort and obtained from University of Ibadan/University College Hospital Research Ethics Committee. A letter of introduction was obtained and issued to the head of department of the selected health facility requesting approval to carry out the study among patients diagnosed with knee osteoarthritis. Informed consent was obtained from the participants before commencing with the study.

\section{Participants and procedure}

Consenting outpatients receiving physiotherapy treatment for knee osteoarthritis were included in the study especially those that had physiotherapy sessions 3 times in a week (Mondays, Wednesdays and Fridays). Patients who were unable to understand written Igbo were excluded from the study.

\section{Validity}

To determine validity, patients recruited were bilingual in English and Igbo. The bilingual participants are native Igbo indigenes from South-easternstern Nigeria and can read and speak English fluently. Patients who had at least two physiotherapy sessions a week were eligible to participate. The time interval was chosen because patients had to fill the English and Igbo versions of the questionnaire in an interval of 2 days.

It took about 5 minutes to complete each questionnaire. Patients who did not attend their subsequent treatment session after filling the first questionnaire were automatically excluded to maintain uniform time interval (example: patients who had sessions on Mondays, Wednesdays and Fridays were recruited. The participants filled the English version of the questionnaire on Monday and the lgbo version on the next therapy session being Wednesday. Patients who filled on Monday but missed treatment on Wednesday were dropped). Fifteen (15) patients completed the study for validity assessment.

As no gold standard exists to the knowledge of the researchers, validity of the b-FMSKOA was expressed in terms of concurrent validity. Spearman's Rho was calculated between the total score of the b-FMSKOA-I (Igbo version) and the total score of b-FMSKOA (English version). Correlation coefficients above 0.6 , between 0.6 and 0.3 , and below 0.3 were considered high, moderate, and low, respectively (Hinkle, Wiersma, \& Jurs, 1998).

\section{Test-retest reliability and validity}

To determine test-retest reliability and validity, the sample of bilingual participants described above completed the lgbo version of the questionnaire again on their third treatment session to assess test-retest reliability.

Reliability was assessed in terms of internal consistency, test-retest reliability. Internal consistency of the b-FMSKOA-I was assessed with Cronbach's alpha using the data from the first administration of the b-FMSKOA-I. In the quality criteria proposed by Terwee et al. (2007) it is suggested that internal consistency of health status questionnaires should be between 0.7 and 0.95 . For test-retest reliability, we calculated the intraclass correlation coefficient (ICC: 2-way random, type agreement) with corresponding 95\% confidence intervals (Cl) between the data from the first and second administration of the b-FMSKOA-I. ICCs above 0.70 were generally considered good (Terwee et al., 2007). 


\section{Results}

\section{Reliability (Test-Retest)}

The internal consistency of the Igbo version of the Brief Fear of Movement Scale for Osteoarthritis using Cronbach's Alpha and the test retest reliability of the translated version of the same scale using Spearman Rank correlation at the level of six items are shown in Table 1.

Table 1

Pearson correlation analysis for the test retests reliability of the Igbo version of Brief Fear of Movement Scale for Osteoarthritis

\begin{tabular}{|lll|}
\hline Items & $\mathbf{R}$ & $\mathbf{p}$ value \\
\hline 1 & 0.945 & $0.000^{\star}$ \\
\hline 2 & 0.943 & $0.000^{\star}$ \\
\hline 3 & 0.932 & $0.000^{\star}$ \\
\hline 4 & 0.441 & 0.131 \\
\hline 5 & 0.905 & $0.001^{*}$ \\
\hline 6 & 0.594 & $0.032^{*}$ \\
\hline
\end{tabular}

A reliability analysis was carried out on Igbo version of Brief Fear of Movement Scale for Osteoarthritis comprising six (6) items. Cronbach's alpha showed the questionnaire to reach acceptable reliability $a=0.85$. Most items appeared to be worthy of retention, resulting in a decrease in the alpha if deleted. The one exception to this was item 4 which would increase the alpha to $a=0.87$. As such, removal of this item should be considered.

Table 1 shows the test retest reliability by testing for correlation of scores between the Igbo version of the Brief Fear of Movement Scale for Osteoarthritis using Spearman correlation. The questionnaire was administered to the participants twice in an interval of 2 days with the aim of testing for reliability as stability. The Pearson correlation coefficient of the variables of interest was within the range of 0.441 and 0.945 with the fourth and first item recording the lowest $(r=0.441)$ and highest $(r=0.945)$ correlation coefficient respectively. All variables showed significant correlation $(p<0.05)$ between the scores obtained from Igbo version of the questionnaires except for the fourth item $(r=0.441 ; p=0.131)$ which showed no significant relationship.

Table 2 shows result of concurrent validity by testing for correlation of scores between the English and Igbo version of the Brief Fear of Movement Scale for Osteoarthritis using Pearson correlation. The correlation coefficient of the variables of interest were within the range of 0.441 and 0.998 and all showed significant correlation $(p<0.05)$ between the scores obtained from the English and Igbo version of the questionnaire except for the fourth item $(r=0.441 ; p=0.131)$ which showed no significant relationship 
Table 2

Pearson correlation analysis for the concurrent validity of the Igbo version of Brief Fear of Movement Scale for Osteoarthritis

\begin{tabular}{|lll|}
\hline Items & $\mathbf{R}$ & $\mathbf{p}$ value \\
\hline 1 & 0.978 & $0.00{ }^{*}$ \\
\hline 2 & 0.943 & $0.000 *$ \\
\hline 3 & 0.926 & $0.000^{*}$ \\
\hline 4 & 0.441 & 0.131 \\
\hline 5 & 0.998 & $0.001 *$ \\
\hline 6 & 0.998 & $0.000 *$ \\
\hline$r=$ Spearman rank correlation coefficient, significant level set at $p<0.05$ \\
\hline
\end{tabular}

Table 3 above shows a correlation matrix displaying how each item of the Igbo version of Brief Fear of Movement Scale for Osteoarthritis correlates to all the other items. Values of I noticed across the diagonal (top left to bottom right) represents instances where the item has been correlated with itself. All the variables with an exception to the fifth item showed a moderate $(r>0.5)$ and significant correlation $(p<0.05)$ with at least two other variables.

Table 3

Inter-item correlation matrix

\begin{tabular}{|c|c|c|c|c|c|c|c|}
\hline Items & & 1 & 2 & 3 & 4 & 5 & 6 \\
\hline \multirow[t]{2}{*}{1} & $\mathrm{R}$ & 1 & .521 & $.670^{*}$ & $.558^{*}$ & .427 & $.597^{\star}$ \\
\hline & p value & & .068 & .012 & .047 & .146 & .031 \\
\hline \multirow[t]{2}{*}{2} & $\mathrm{R}$ & .521 & 1 & $.866^{\star \star}$ & .371 & .449 & $.610^{*}$ \\
\hline & $\mathrm{p}$ value & .068 & & .000 & .213 & .124 & .027 \\
\hline \multirow[t]{2}{*}{3} & $\mathrm{R}$ & $.670^{*}$ & $.866^{\star \star}$ & 1 & .282 & .484 & $.641^{\star}$ \\
\hline & $p$ value & .012 & .000 & & .350 & .094 & .018 \\
\hline \multirow[t]{2}{*}{4} & $\mathrm{R}$ & $.558^{*}$ & .371 & .282 & 1 & .021 & $.676^{*}$ \\
\hline & $\mathrm{p}$ value & .047 & .213 & .350 & & .946 & .011 \\
\hline \multirow[t]{2}{*}{5} & $\mathrm{R}$ & .427 & .449 & .484 & .021 & 1 & $.664^{*}$ \\
\hline & $p$ value & .146 & .124 & .094 & .946 & & .013 \\
\hline \multirow[t]{2}{*}{6} & $\mathrm{R}$ & $.597^{\star}$ & $.610^{*}$ & $.641^{\star}$ & $.676^{*}$ & $.664^{*}$ & 1 \\
\hline & $p$ value & .031 & .027 & .018 & .011 & .013 & \\
\hline
\end{tabular}

\section{Discussion}

The aim of this study was to translate the b-FMSKOA scale into Igbo (b-FMSKOA-I) and to study its validity and reliability in order to investigate psychometric properties among lgbo-speaking patients with knee osteoarthritis. The results indicate good validity and reliability for the b-FMSKOA-I scale.

\section{Validity}


The b-FMSKOA consists of only six items. The scale is considered one-dimensional because factors with less than five items are generally considered weak and unstable (Costello \& Osborne, 2005). The exploratory factor analysis supported the one-factor structure, so the scale cannot be separated.

Concurrent validity was assessed by correlating the results of both English and Igbo versions of the b-FMSKOA. To our knowledge, these two questionnaires have not been used before to test hypotheses on concurrent validity. Our predefined hypotheses were therefore primarily based on previous research comparing other translated versions of questionnaires related to fear of movement. We found a high correlation between the b-FMSKOA and the b-FMSKOA-I. This met our expectations, as both questionnaires measure same construct except in different languages. However, there are some differences. The correlation coefficient of the variables of interest were within the range of 0.441 and 0.998 and all showed significant correlation $(p<0.05)$ between the scores obtained from the English and Igbo version of the questionnaire except for the fourth item $(r=0.441 ; p=0.131)$ which showed no significant relationship.

Item 4 stated "Naani ido anya ka m ghara iga ije na ekwesighi ekwesi bu ihe kachasi mma m nwere ike ime were gbochie mgbu $m$ ikawanye njo" and "Simply being careful that I do not make any unnecessary movement is the safest thing I can do to prevent my pain from worsening". The keyword here is 'safest' which was translated as 'Kachasi mma' which could mean 'best' or 'safest' on its own, but was translated as 'best' by most respondents leading to the negative correlation. From patient's perspective, the question alone already has a negative correlation, as it is not the best translation of the intended statement, and as such, the result gotten. Therefore, rephrasing or removal of this item should be considered or a different approach to administration of the questionnaire where the ambiguity would be erased.

\section{Reliability and stability}

Internal consistency of the b-FMSKOA-I was good with a Cronbach's alpha score of $a=0.85$. Most items appeared to be worthy of retention, resulting in a decrease in the alpha if deleted. The one exception to this was item 4 which would increase the alpha to $a=0.87$. As such, removal of this item should be considered.

Test-retest reliability of the I-PRRS-NL scale was good, with Pearson correlation coefficient of the variables of interest was within the range of 0.441 and 0.945 with the 4 th and 1 st item recording the lowest $(r=0.441)$ and highest $(r=0.945)$ correlation coefficient respectively. All variables showed significant correlation $(p<0.05)$ between the scores obtained from Igbo version of the questionnaires except for the fourth item ( $r=0.441 ; p=0.131)$ which showed no significant relationship. As these different reliability tests have not been previously investigated, no comparison with literature could be made. However, the b-FMSKOA-I as a whole without, considering the different variables, shows a significant correlation with each other and therefore strongly indicates that the b-FMSKOA-I may be a useful research tool like its English counterpart.

The minimal important change of the b-FMSKOA-I scale has not yet been determined, hence further research is needed to evaluate responsiveness (sensitivity to change) and MIC of the b-FMSKOA-I as patients undergo treatment for knee osteoarthritis.

\section{Conclusion}

The b-FMSKOA was successfully translated into Igbo; the b-FMSKOA-I. The b-FMSKOA-I scale showed sufficient validity and reliability to assess fear of movement for individuals living with knee osteoarthritis. Because of the recommendation from the developer on all six items of the scale, only the total score should be used. The b-FMSKOA-I can therefore, be used in cross-sectional studies and can be used in clinical settings to effectively and accurately assess fear of movement in patients with knee OA. It can also be used as an outcome measure during treatment.

\section{Declarations}

\section{Funding}

This study was self-funded.

\section{Conflicts of interest}

None declared.

\section{Ethical approval:}




\section{References}

1. Hanrungcharotorn, U., Pinyopasakul, W., \& Pongthavornkamol, K. (2017). Factors Influencing Physical Activity among Women with Osteoarthritis of the Knee. Pacific Rim International Journal of Nursing Research, 21(1), 5-17. Retrieved from https://www.ncbi.nlm.nih.gov/pmc/articles/PMC4405030/pdf/nihms680923.pdf\%0Ahttp://content.wkhealth.com/linkback/openurl? sid=WKPTLP:landingpage\&an=00002281-201505000-00012

2. The Royal Colleague of Orthopaedic Surgeon of Thailand. The guideline of knee osteoarthritis 2011 [cited 201418 October]. Available from: .

3. Vlaeyen JW, K.-S. A. (1995). The role of fear of movement/(re)injury in pain. Journal of Occupational Rehabilitation, 5:235-52.

4. Dekker J, Tola P, Aufdemkampe G, Winckers M. Negativeaffect, pain and disability in osteoarthritis patients: the medi-ating role of muscle weakness. Behav Res Ther 1993; 31:203-6.

5. Somers TJ, Keefe FJ, Pells JJ, Dixon KE, Waters SJ, Riordan PA,et al. Pain catastrophizing and pain-related fear in osteoarthritis patients: relationships to pain and disability. J Pain Symp-tom Manage 2009;37:863-72

6. Shelby, R. A., Somers, T. J., Keefe, F. J., Devellis, B. M., Patterson, C., Renner, J. B., \& Jordan, J. M. (2012). Brief fear of movement scale for osteoarthritis. Arthritis Care and Research, 64(6), 862-871. https://doi.org/10.1002/acr.21626

7. Beaton DE, Bombardier C, Guilleman F, Ferraz MB (2000): Guidelines for the process of Cross-Cultural adaptation of Self-Report Measures. Spine 25: 3156-3191.

8. Babajide OA (2001): Language attitude patterns of Nigeria in Language attitude and language conflict in West Africa Igboanusi $\mathrm{H}$. Eni crownfit Publisher S. Ibadan. 1-13.

9. Akinpelu AO, Maruf FA, Adegoke BOA (2006): Validation of a Yoruba translation of the World Health Organizationखs quality of life scale-short form among stroke survivors in Southwest Nigeria. African Journal of Medicine and Medical Science. 35:417-424.

10. Hinkle, D. E., Wiersma, W., \& Jurs, S. G. (1998). Applied statistics for the behavioral sciences. Boston, MA: Houghton MiZin.

11. Terwee, C. B., Bot, S. D., de Boer, M. R., van der Windt, D. A., Knol, D. L., Dekker,J., ... de Vet, H. C. W. (2007). Quality criteria were proposed for measurement properties of health status questionnaires. Journal of Clinical Epidemiology, 60, $34-42$.

12. Costello, A. B., \& Osborne, J. W. (2005). Best practices in exploratory factor analysis: Four recommendations for getting the most from your analysis. Practical Assessment, Research and Evaluation, 10, 7.

\section{Figures}




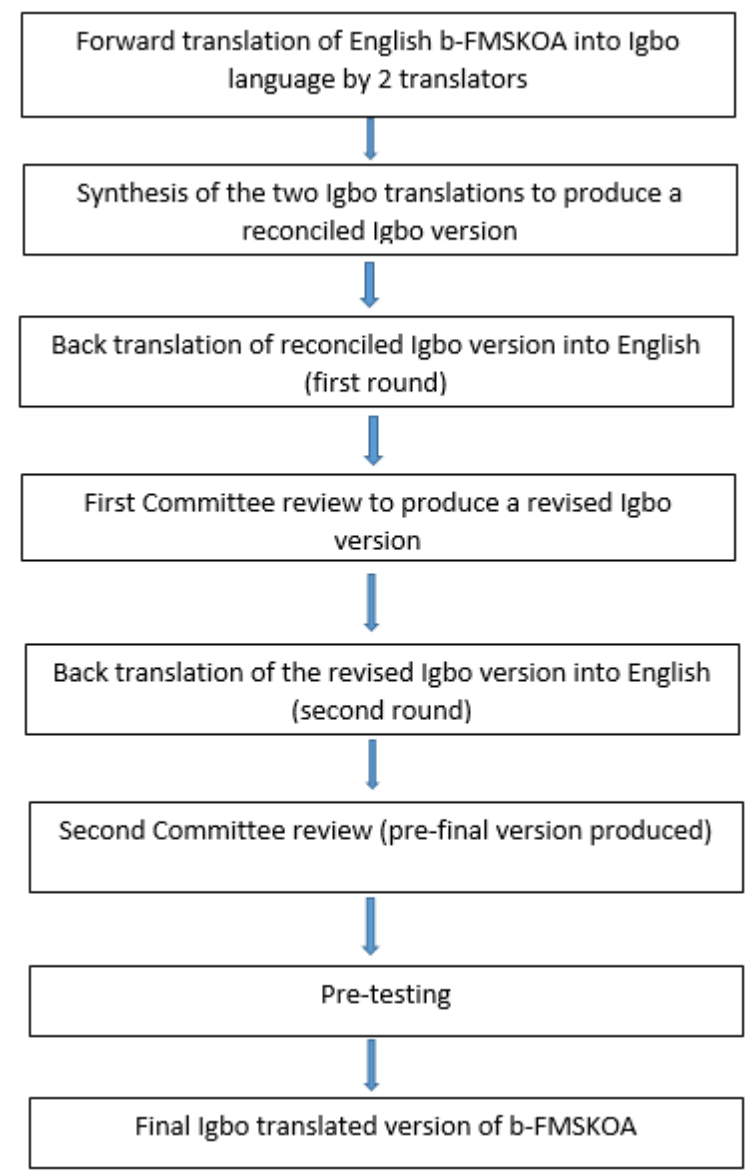

Figure 1

Flow chart of the steps involved in the translation of b-FMSKOA into Igbo language

\section{Supplementary Files}

This is a list of supplementary files associated with this preprint. Click to download.

- APPENDIX.docx 